Cahiers de philosophie de l'université de

\title{
Quelques remarques à propos du problème de la réalité
}

Roland Omnès

\section{(2) OpenEdition}

1 Journals

Édition électronique

URL : https://journals.openedition.org/cpuc/1382

DOI : $10.4000 /$ cpuc.1382

ISSN : 2677-6529

Éditeur

Presses universitaires de Caen

\section{Édition imprimée}

Date de publication : 15 décembre 2008

Pagination : 259-268

ISBN : 978-2-84133-332-5

ISSN : 1282-6545

\section{Référence électronique}

Roland Omnès, "Quelques remarques à propos du problème de la réalité », Cahiers de philosophie de l'université de Caen [En ligne], 45 | 2008, mis en ligne le 04 septembre 2020, consulté le 05 février 2023. URL : http://journals.openedition.org/cpuc/1382 ; DOI : https://doi.org/10.4000/cpuc.1382

\section{(c) (7) (8)}

Creative Commons - Attribution - Pas d'Utilisation Commerciale 4.0 International - CC BY-NC 4.0 https://creativecommons.org/licenses/by-nc/4.0/ 


\section{QUELQUES REMARQUES À PROPOS DU PROBLÈME DE LA RÉALITÉ}

Un certain temps s'est écoulé depuis l'agréable rencontre qui s'est tenue à Caen, si bien que les idées émises alors par certains d'entre nous ont pu évoluer ou perdre de leur actualité. En ce qui me concerne, il se trouve que la partie essentielle de ma propre contribution d'alors a été publiée dans l'intervalle ${ }^{1}$ et, en outre, diverses réflexions m'ont conduit depuis à considérer la question de la réalité sous un nouveau jour. Certaines de ces idées sont encore au stade de discussions avec des collègues, qui peuvent les préciser ou les corriger. Des erreurs peuvent encore s'y trouver, graves ou légères, mais il est également possible qu'elles modifient substantiellement les données de ce problème déjà ancien. C'est pourquoi il m'a paru préférable d'éviter ici le style académique habituel pour adopter un ton plus personnel et dire ce que c'est que de vivre, dans l'intimité, en compagnie du problème de la réalité et de son retour récurrent quand on croyait l'avoir mis de côté. En effet, s'il est vrai que le «moi» soit haïssable quand il s'affirme de façon péremptoire, le «je» permet en revanche de faire apparaître des esquisses d'options, des espoirs d'ouverture, des perspectives qui semblent d'autant plus séduisantes qu'elles sont encore changeantes, comme l'esprit du chercheur.

\section{Remarques anciennes}

Il se trouve que ce problème de la réalité fut l'occasion pour moi d'entrer "en philosophie». Cela remonte à un peu plus d'une douzaine d'années et j'avais eu la chance de participer à une sorte de renaissance qui venait de se produire dans l'interprétation des lois

1. Omnès 2006 . 
quantiques. J'avais un peu contribué aux trois principaux domaines où le mouvement avait eu lieu. L'un d'eux était l'émergence de la physique classique à partir des lois quantiques, d'où le déterminisme classique apparaissait comme un cas particulier remarquable du probabilisme quantique (c'est-à-dire comme une propriété dont la probabilité d'erreur est singulièrement faible). Un autre était le retour à la logique standard grâce aux «histoires de Griffiths" qui apportaient une liaison cohérente entre l'intuition empirique et la forme mathématique des lois (Griffiths a montré récemment comment les paradoxes divers qui semblaient obérer la physique se dissipent ainsi ${ }^{2}$ ). Il y avait eu enfin la grande aventure de la décohérence d'où le chat de Schrödinger sortait vraiment vivant ou mort et non plus les deux à la fois. Mais quels changements ces découvertes pouvaient-elles entraîner en philosophie des sciences? Telle fut alors la question qui s'empara de moi et qui devint le centre de mon tout premier livre de "philosophie" ${ }^{3}$.

D'autres ont écrit des pages du même genre, souvent meilleures $^{4}$, mais la manière dont je vécus alors le problème de la réalité se résumait en quelques grands traits où il apparaissait grandiose et profond, fascinant. Les avancées dont je viens de faire état avaient en effet toutes trois un point commun: elles s'appuyaient directement sur les principes de la mécanique quantique, tels qu'ils furent dégagés avant 1930 et qu'ils demeurent encore, comme s'ils contenaient en eux-mêmes tous les éléments nécessaires à leur propre interprétation. Il suffisait de dégager les concepts qui s'y tenaient cachés par une analyse serrée et l'on constatait alors que des moyens mathématiques puissants (issus de l'analyse microlocale) extrayaient la physique classique du substrat quantique, que la construction des histoires de Griffiths s'appuyait directement sur les principes qui attiraient l'attention sur le langage adéquat à l'interprétation. Enfin, la décohérence résultait d'une étude plus attentive des conditions réelles d'une expérience, en tenant compte surtout de ce qui n'y est pas mesuré (et qui ne peut pas l'être). Cette triple voie d'approche, dégageant les germes d'une interprétation philosophique à partir des principes abstraits, semble de plus en plus gagner du terrain, mais elle ne s'est pas faite sans mal et je me souviens par exemple d'une conversation que j'avais eue en 1988 avec John Bell et au cours

2. Griffiths 2002 .

3. Omnès 1994.

4. d'Espagnat 1994 et d'Espagnat 2002. 
de laquelle je lui présentai la perspective de cette idée. Amicalement, mais fermement, il me dit qu'à son avis, cette voie était impossible.

Elle allait se montrer pourtant féconde et son attrait s'est beaucoup renforcé depuis. Elle permet de déduire les règles de Copenhague régissant les mesures, comme autant de conséquences directes des principes de base. Elle offre aussi une image harmonieuse de la connaissance en physique en la faisant reposer de manière égale sur l'expérience et sur la cohérence logique. Pourtant, cet idéal est encore loin d'être acquis. La correspondance entre la réalité et la théorie serait parfaite en effet, si on ne voyait apparaître au tout dernier moment un gouffre infranchissable qui les sépare. C'est en ce vide béant que réside le "problème de la réalité ", dit encore "problème de l'unicité" et rien n'est plus facile que de le formuler: La mécanique quantique se fonde sur le hasard et c'est donc une théorie statistique, dans son essence. En tant que telle, elle ne peut prédire que des possibilités et leur attribuer des probabilités, de sorte que rien ne lui est plus étranger, du moins en apparence, que l'unicité du Réel. Or on sait que des philosophes (comme Wittgenstein dans ses Investigations Philosophiques) voient dans cette unicité le caractère essentiel de la réalité. Ainsi, quand le maître maçon désespère de faire comprendre à son apprenti ce qu'est une brique, il ne peut qu'en montrer une du doigt en disant: " cela!", or cette définition grâce à une brique réelle n'a de sens que si "cela" est unique. En outre on sait qu'en philosophie des sciences, l'unicité du réel - c'est-à-dire des faits - est la base absolue de la méthode expérimentale, si bien que lorsqu'on constate que les principes quantiques s'écartent de cette unicité, tout l'édifice de la connaissance tremble sur ses bases.

En essayant de dégager au fil des années les abords de ce problème, il m'apparut que certains aspects d'ordre philosophique y avaient été négligés. Ainsi, je suis mal à l'aise quand je lis, encore et encore, des comparaisons plus ou moins futiles entre les degrés de vraisemblance des univers parallèles d'Everett, des variables cachées sous la forme que Bell leur a donné ou sous celle de Bohm, ou encore de la réduction spontanée à la manière de Ghirardi, Rimini et Weber. Tout cela me semble insupportablement scolastique. Les propositions qui furent à l'origine de ces idées et les recherches qu'elles entraînèrent étaient incontestablement légitimes et il est bon d'essayer d'en repousser les limites, mais il serait bon aussi qu'on en cherche d'autres et je trouve absurde qu'on prétende parfois choisir entre elles sur des bases philosophiques, comme au bon vieux temps scolastique de la méthode d'élimination. 
La seule valeur qu'on puisse accorder à la philosophie dans ce domaine est sans aucun doute celle de la Critique, laquelle comporte avant tout la capacité de suspendre son jugement. En revanche, je voudrais aussi signaler certaines limites dans lesquelles il me semble que cette Critique se cantonne trop, quand il n'arrive pas qu'elle s'y fourvoie. Ainsi, lorsqu'on discute ces questions, on souligne toujours l'existence du hasard quantique et l'on s'en tient souvent là. Mais cette description est à courte vue et s'en tient aux apparences. Elle fait bon marché d'une connaissance qui fut apportée par Feynman et qui date de plus d'un demi-siècle, avec les histoires qui portent son nom. Il montra alors clairement que les amplitudes quantiques englobent une immense multiplicité de possibles. Tout mouvement d'une particule est un possible, quelles que soient sa trajectoire et les variations de sa vitesse; tous ces "chemins", ces "histoires", ont pour amplitudes quantiques des nombres complexes qui ont un même module (la même grandeur) pour toutes les histoires et qui ne diffèrent que par leurs phases. Tous les chemins contribuent de manière égale à l'évolution selon l'équation de Schrödinger et l'amplitude de probabilité finale résulte de l'addition de leurs amplitudes, toutes de même poids mais se conjuguant ou s'annulant réciproquement par interférence, c'est-à-dire se superposant. Le principe de superposition est ainsi sans aucun doute l'aspect le plus profond des lois quantiques. Mais c'est aussi l'obstacle majeur qui s'oppose à penser les lois de façon conventionnelle, c'est-à-dire au travers d'une conception trop banale de la philosophie de la connaissance.

Toute la construction du modèle standard des leptons et des quarks a montré la supériorité théorique et pratique des conceptions de Feynman sur les formulations antérieures. Penser la nature comme une superposition de tous les possibles s'est ainsi révélé plus fécond que l'idée plus ancienne d'une fonction d'onde, qui ne peut exprimer que le hasard. Or cette constatation n'a pas vraiment pénétré la philosophie des sciences, parce que notre cerveau $y$ fait obstacle. Ce cerveau, issu de l'évolution de notre espèce et de celles qui ont précédé, est ainsi fait qu'il ne peut concevoir intuitivement qu'une réalité unique, que des phénomènes continus, et il ne peut imaginer ou décrire par ses mots que des éventualités distinctes, bien séparées et mutuellement exclusives ${ }^{5}$. Or, pour autant que je sache, la philosophie ne s'aventure pas au-delà de la logique

\section{Cf. Omnès 2006.}


modale dans sa conception du possible et n'aperçoit que de loin la falaise abrupte du principe de superposition. Son langage ne le lui permet pas, car son vocabulaire reste marqué par des associations trompeuses et limité par une grammaire causale, adéquate au seul monde macroscopique. Ce n'est qu'au moyen des mathématiques que le cerveau humain parvient à exprimer et à exploiter un des principes philosophiques les plus profonds de la nature : le principe de superposition.

Des réflexions de ce genre conduisent inévitablement à revisiter la philosophie des mathématiques ${ }^{6}$. La première constatation qu'on peut faire dans ce domaine est un extraordinaire manque de recouvrement entre la philosophie des mathématiques et celle de la physique. Pourtant, tout invite à les comparer. Leurs "crises" ont été simultanées, mais elles n'ont fait que les séparer. Les mathématiques se sont éloignées de leurs origines naturalistes lorsqu'elles ont constaté que leurs objets devenaient nécessairement plus abstraits que les quantités "physiques" rencontrées dans la nature. Or, à l'époque même où l'on s'interrogeait sur la nature des mathématiques, la découverte des quanta et les interrogations qui suivirent ne permettaient plus de s'appuyer sur le socle de la réalité physique. Il n'y eut plus alors vraiment de philosophie des sciences exactes, mais tout au plus une histoire de celles-ci, accompagnée d'une collection de philosophies séparées et diverses. Pourtant, depuis, beaucoup des objets bizarres, des "ornithorynques" mathématiques devant lesquels Hermite se voilait la face, sont réapparus en physique. On doit désormais les prendre en compte pour raisonner avec rigueur sur les espaces d'Hilbert de la théorie quantique et la physique en a découvert de nouveaux (les histoires de Feynman, par exemple, semblent à l'étroit dans les limites de la théorie des ensembles). Quand on compare en outre les sciences physique et mathématique au niveau de leurs axiomes, on constate que ceux qui sont indispensables aux mathématiques de la physique actuelle engendrent tout aussi bien le temple tout entier des mathématiques contemporaines. La parenté des problèmes concernant la nature des mathématiques et ceux de l'existence des lois physiques devient de plus en plus manifeste. Quel plus beau champ de pensée pourrait tenter un philosophe épris de grands espaces? Mais on y rencontre peu de monde.

6. Cf. Omnès 2005. 


\section{Remarques de dernière heure}

Il est rare qu'on écrive sur une théorie en train de se faire alors qu'elle est encore fragile, peut-être éphémère. C'est pourtant ce qu'Ivahn Smadja m'a encouragé à faire, alors que je me sentais écartelé entre le désir de tenir ma parole en livrant un texte depuis longtemps promis et le sentiment que mes idées étaient devenues différentes depuis, et encore en cours d'évolution. Personnellement, je suis toujours resté insatisfait des "solutions" proposées au problème de la réduction. Je ne dédaigne aucunement les efforts et l'intelligence de leurs concepteurs, mais je constate que les idées d'Everett ou de Bohm ont plus de cinquante ans, celles de Bell plus de quarante, celles de Ghirardi, Rimini et Weber ont un quart de siècle et rien de nouveau ne se passe. Les congrès et les publications ressassent indéfiniment des commentaires inchangés, pareils à des gloses classiques comparant des doctrines antiques.

J'ai moi-même autrefois cédé à ces sirènes, du moins à celles qui chantent en philosophie, en suggérant que, peut-être, la béance quasi parfaite qui séparait la théorie quantique de l'unicité du Réel marquait une limite absolue du programme cartésien (ainsi que le nommait Husserl). Peut-être que tout ne s'exprimait pas par des formes mathématiques dans la nature. J'ai renoncé depuis à cette idée car elle ne pouvait que se refermer sur son renoncement, sans aucune fécondité.

J'ai dit plus haut que les progrès des dernières décennies en matière d'interprétation furent dus à part égale aux travaux des expérimentateurs et des théoriciens. Chez ces derniers, trois idées se sont révélées fécondes : la décohérence, la dérivation de la physique classique et la remise en place de la logique dans les histoires cohérentes de Griffiths. Toutes trois s'appuient directement sur les principes quantiques, comme si ceux-ci contenaient en eux-mêmes tous les concepts nécessaires à leur propre interprétation, mais à une exception près de très grande taille: la fameuse réduction de la fonction d'onde, ou la "réduction» de manière plus générale. Quant à moi, peu à peu, le plaisir insistant de méditer sur la nature des lois me conduisait à prendre une idée simple de plus en plus au sérieux: Se pourrait-il que tout résulte des principes, y compris la réduction? Je constatai que les raisons qu'on donnait traditionnellement à l'encontre de cette éventualité n'étaient guère à la hauteur de l'enjeu: un peu légères, disons.

En restant dans le registre de la confession, j'ajouterai que j'ai conscience à la fois de l'âge que j'ai atteint et d'une certaine chance 
à pouvoir exercer encore ma réflexion. L'âge me dissuade de croire que je pourrais contribuer à résoudre ce grand problème, alors que le plaisir de penser le ramène sans cesse devant mes yeux. J'ai formidablement envie de connaître la réponse avant de rencontrer Saint Pierre ou le porte-clef du silence. D'où la suite.

Il m'a semblé que la question était si grande que sa réponse devait être simple, mais en rompant avec nos habitudes de pensée. Quoi de plus simple alors que d'observer que l'unicité de la réalité imprègne tout ce qui nous entoure, à notre échelle, et aussi que toutes les données de la physique concourent à la nier au niveau des atomes? Donc, tout se passe quasiment sous nos yeux, à la limite commune au quantique et au classique, très loin des particules réelles et inaccessibles de Bohm, là où tout apparaît connu, sans variables cachées mystérieuses, de manière très concrète et non dans une métaphysique délirante d'univers multiples de sciencefiction. Dans la chose elle-même, pourrait-on dire.

J'ai beaucoup tourné en rond sans vraiment chercher à résoudre le problème, mais en le gardant en bouche, pourrait-on dire. Je passe sur les promenades intellectuelles où cela me conduisait, souvent rêveuses ou parfois arrêtées par des équations difficiles. Jusqu'au jour où j'ai cru deviner où la bête gîtait. Ce ne pouvait être que sous nos yeux. En glissant sur les aspects techniques, on peut dire que c'est encore une fois au niveau de la frontière indécise entre le quantique et le classique. Or il se trouve que ce domaine m'est familier et j'y ai donné par exemple une explication du déterminisme et de ses conditions de validité, comme autant de conséquences des lois quantiques. Et je n'ai rien vu! Personne, pour autant que je sache, n'a rien vu. On n'a pas vu que le principe de correspondance de Bohr nous aveuglait en mettant tout le poids de la pensée sur la transition quantique-classique, sur le fameux "principe de correspondance" où on ne s'intéresse toujours qu'à un objet macroscopique qui est donné. On ne s'interroge pas sur la génération de la classicité quand un tel objet apparaît, que ce soit une bulle dans une chambre à bulles, une gouttelette dans une chambre de Wilson, une étincelle dans un compteur Geiger ou une chambre à fils, un signal électrique quelque part. On n'a pas examiné non plus la stabilité de la réalité classique.

Pour qui sait la difficulté des recherches qui ont conduit à ce que l'on sait, il apparaît néanmoins aussitôt que ces problèmes sont redoutables, d'un point de vue technique. Mais peu à peu, en y songeant, il m'apparut qu'on avait sous-estimé l'importance de la structure classique de la réalité. Il est vrai que les lois classiques 
émergent des lois quantiques dans des conditions macroscopiques, mais il existe un effet réciproque. Le monde classique des objets macroscopiques (qui commence avec quelques dizaines d'atomes) définit un cadre d'action phénoménologique pour les lois quantiques. Je m'explique : une cellule, vivante ou morte, a une certaine forme et celle-ci contraint les fonctions d'onde de ses atomes intérieurs. Elle reçoit la lumière du soleil, elle émet pendant la nuit un rayonnement infrarouge, elle baigne dans un milieu où tout est classique, ou presque, où tout se répond classiquement, même si cette structure classique a sa source dans des lois quantiques. D'un point de vue méthodologique, on pourrait dire que la réflexion a beaucoup porté jusqu'ici sur la structure des lois et sur leur expression mathématique aux niveaux quantique et classique, mais peu sur les conditions initiales et les conditions aux bornes que la réalité classique impose par rétroaction au monde quantique.

À dire vrai, cette vision simple ne m'est apparue qu'après coup, mais elle constitue cependant un point de départ vraisemblable vers le bel et redoutable problème de la réduction. Il y eut auparavant une longue période de tâtonnements où je cherchais à associer de près réduction et décohérence. Une conclusion étonnante en résultait. Pour des raisons d'ordre de grandeur, il semblait impossible de concilier la réduction avec les principes quantiques de base, à moins que l'univers ne soit dans un état pur ou qu'en d'autres termes, il soit décrit dans sa totalité par une fonction d'onde. L'idée elle-même n'est pas nouvelle. Elle fut introduite en 1983 par Hartle et Hawking en réponse à un grand problème de cosmologie, celui de l'extraordinaire degré d'ordre des débuts de l'univers. J'allais la retrouver plus tard, toujours pour des raisons d'ordre de grandeur, et je poursuis maintenant l'histoire en admettant cette hypothèse.

On a beaucoup invoqué un certain caractère non linéaire de la réduction, qui paraissait incompatible avec le principe de superposition en excluant ainsi la cohérence étroite des bases quantiques avec la réduction. J'avais toujours douté de cet argument brutal et une étude plus attentive me conduisit à trouver l'erreur. Ce qu'on sait déjà permet de montrer que les propriétés classiques sont contenues dans la fonction d'onde, c'est-à-dire lui appartiennent. Or quand on explicite cette relation, on constate qu'elle se traduit par une équation non linéaire à laquelle la fonction d'onde se plie. En outre, ce genre de propriété non linéaire ne contredit nullement le principe de superposition, car la fonction d'onde de l'univers est unique et ne se superpose à aucune autre. En d'autres termes, le principe de superposition, qui s'applique parfaitement à un sous-système 
de l'univers, appartient au domaine des lois. Il ne s'applique pas à la totalité de l'univers, car celui-ci constitue sa propre référence. On peut prendre un exemple analogue en considérant l'invariance relativiste des lois de la physique; cette invariance s'exprime par des transformations linéaires (les transformations de Lorentz), mais aucune linéarité ne s'applique aux phénomènes terrestres ordinaires, car la Terre définit sa propre référence.

Un peu d'analyse mathématique montre alors que, dans une expérience de mesure quantique, les relations de "classicité" précédentes sont brisées. La description habituelle d'une telle expérience, où l'on pose que la superposition linéaire de deux états quantiques se transmet en une superposition des appareillages, n'a plus alors aucun sens. En fait, une telle superposition est dénuée de sens, car elle ne peut s'exprimer mathématiquement sans détruire la classicité. On voit alors poindre la signification de la mesure et de la réduction: il s'agirait, pour la première, d'une rupture des caractères classiques et, pour la seconde, de leur rétablissement.

Au moment où j'écris, je dispose depuis quelques mois d'un modèle qui rend compte apparemment de la réduction. Il repose entièrement sur les premiers principes et conduit à une conclusion étonnante: ou bien l'univers était initialement dans un état quantique pur et la réduction a bien lieu, y compris dans ses aspects quantitatifs, ou bien l'état de l'univers est désordonné et il faut alors abandonner l'espoir de voir la réduction émerger de ces seuls principes. Que dire de plus? Ces réflexions sont encore enfermées dans un emballage technique, aussi bien physique que mathématique, et il faudra du temps pour les en dégager et faire apparaître leur substance de façon claire, dans un langage plus simple. Il serait aussi vain que prématuré de commenter les perspectives philosophiques qu'il laisse entrevoir, ainsi que d'autres plus physiques. Tout peut tomber à bas si une erreur, une faille, y apparaît après un peu plus de travail. Aussi me contenterai-je de dire la fascination qu'on éprouve à plonger plus loin dans les lois de la nature pour les faire parler, pour leur arracher leurs secrets et mettre leur cohérence à l'épreuve. Que ce travail se termine par un nouvel édifice ou par un amas de décombres, cela importe peu au fond pour le chercheur qui aura appris quelque chose de toute manière et pourra dire, comme le toujours jeune Rimbaud: «Cela, c'est fini, je sais maintenant saluer la beauté».

Roland OMNÈs Laboratoire de Physique Théorique, Université de Paris-Sud 


\section{Références bibliographiques}

D’Espagnat B. (1994), Le réel voilé, analyse des concepts quantiques, Paris, Fayard.

D'Espagnat B. (2002), Traité de physique et de philosophie, Paris, Fayard. GRIFFITHS R. (2002), Consistent Quantum Theory, Cambridge, Cambridge University Press.

OMNÈs R. (1994), Philosophie de la science contemporaine, Paris, Gallimard.

OMNÈs R. (2005), Converging realities, toward a common philosophy of mathematics and physics, Cambridge, Cambridge University Press.

OMNÈs R. (2006), Les indispensables de la mécanique quantique, Paris, Odile Jacob. 